\title{
Character Association among Cane Yield and their Component Traits in Sugarcane under Waterlogged Condition
}

\author{
Bal Krishna* and Dharm Nath Kamat \\ Department of Plant Breeding and Genetics, Sugarcane Research Institute, Dr. Rajendra Prasad \\ Central Agricultural University, Pusa, Samastipur, Bihar 848 125, India \\ *Corresponding author
}

\section{A B S T R A C T}

\begin{tabular}{|l|}
\hline Ke y w o r d s \\
$\begin{array}{l}\text { Sugarcane, } \\
\text { Correlation } \\
\text { coefficient, } \\
\text { Waterlogging. }\end{array}$ \\
\hline Article Info \\
\hline $\begin{array}{l}\text { Accepted: } \\
\text { 21 September } 2017 \\
\text { Available Online: } \\
\text { 10 October } 2017\end{array}$ \\
\hline
\end{tabular}

A study was conducted with sixteen clones received from Sugarcane Research Institute, D.R.P.C.A.U., Pusa, Samastipur, Bihar. The result of present study clearly indicated that Cane yield ( $\mathrm{t} / \mathrm{ha}$ ) had significant and positive correlation with germination percentage at $45 \mathrm{DAP}$, number of shoots at $120 \mathrm{DAP}(000 / \mathrm{ha})$, number of shoots at 240 DAP (000/ha), number of millable canes at harvest (000/ha), sugar yield (t/ha), plant height at $240 \mathrm{DAP}$, plant height at $360 \mathrm{DAP}$, single cane weight, cane diameter at harvest, leaf area index before waterlogging, leaf area index 30 days after waterlogging, leaf area index 60 days after waterlogging, number of fully emerged leaves at 30 days after waterlogging and number of fully emerged leaves at 60 days after waterlogging. Thus, these traits play a greater role as yield contributing characters.

\section{Introduction}

A considerable area under sugarcane crop in several parts of India (Assam, Bihar, and West Bengal, eastern Uttar Pradesh, Coastal region of Andhra Pradesh, Tamil Nadu, Kerala and Karnataka) are exposed to stagnant water for two to three months during monsoon season. The short fall in yield potential is mainly due to various biotic and abiotic stresses in Bihar in which waterlogging is the main factor because in Bihar about 35-40 per cent of sugarcane remains waterlogged during monsoon season which coincides with the grand growth period of the crop. To enhance the productivity of this crop in Bihar, there is need to identify such type of sugarcane variety/clone which have ability to tolerate waterlogging condition. For breeding purposes, in order to develop improved waterlogged tolerant varieties, well planed breeding efforts are required. In this crop, varietal improvement requires very specific photothermal period for flowering and viable seed setting.

Due to unfavorable environment condition Sugarcane does not flower profusely in most of the cane growing area in the world. This limitation in cane flowering and viable seed production has narrowed genetic base. Various traits are accounted for variations in cane and sugar yields. Many of these characters components are quantitatively 
inherited and inter-related with each other. Knowledge of inter-relationship among the various characters is considered to be important in devising proper selection strategies in a sugarcane breeding De Sousa and Scott (2005), Stevenson (1965) pointed out that there may not be specific genes controlling the complex characters, but sum total effect of its component might be influencing the two most important characters, namely, cane yield and sucrose in sugarcane. Correlation coefficient analysis has been extensively used by plant breeders to obtain precise information on interrelationship among plant traits to better assess outcome of selecting one or more trait Furtado et al., (2002). The knowledge of association of cane and sugar yields with other traits helps breeders select suitable and desirable plant type.

In multiple objective selections, correlation between characters in varying environmental conditions is of immense importance for overall response to selection. Pattern of association existing in the yield and quality components in subtropical environment has not been thoroughly examined over the years in this crop Tyagi et al., (2012). The result of present research will be helpful in planning future breeding programme for the evaluation of high yielding and better quality sugarcane varieties under waterlogging condition in Bihar. The overall scope of study was to enhance the efficiency of selection. Correlation coefficient of yield contributing characters has always been helpful as basis for selection in breeding programs.

\section{Materials and Methods}

\section{Climate}

The experimental plot was well leveled but remains waterlogged during May to September 2016 due to low land. The average depth of water varied from 30 to $90 \mathrm{~cm}$ and $130 \mathrm{~cm}$ for 15 days during September, 2016. The month wise temperature, relative humidity and rainfall data has been provided at Figure 1.

\section{Treatments and experimental design}

The sixteen sugarcane clones viz. CoP 09437, CoP 11439, CoP 11440, CoP 12438, CoP 12439, CoP 13438, CoP 13439, CoP 14438, CoP 14439, CoP 15439, CoP 15440, CoP 15441, BO 155 and CoP 2061 were evaluated along with two standard BO91 and BO154. The trial was laid out in randomized block design with three replications. Equal number of three budded sets of each clone was planted.

\section{Data collected and analysis}

Data were collected for yield attributing traits viz. germination percentage at 45 DAP (Days After Planting), number of shoots at 120 DAP. (000/ha), plant height at 150, 240, 360 days $(\mathrm{cm})$, number of fully emerged leaves at 30 days and 60 days after waterlogging, leaf area index before waterlogging, at 30 and 60 days after waterlogging, number of nodes with aerial roots, cane diameter at harvest (cm), number of shoots at 240 DAP (000/ha), number of millable canes at harvest (000/ha), single cane weight $(\mathrm{Kg})$, cane yield ( $\mathrm{t} / \mathrm{ha}$ ) and sugar yield (CCS t/ha) at harvest and juice quality traits viz. brix, Pol and Purity at 10 \&12 months stage (\%), CCS \% at 10 and 12 months stage. Chemical analyses of sugarcane juice for brix (\%), pol (\%), purity (\%) and CCS (\%) were done.

\section{Correlation}

Genotypic and phenotypic correlation coefficients among the characters under study were estimated according to the statistical techniques outlined by Al-Jibouri et al., (1958) as well as Panse and Sukhatme (1967). 


\section{Results and Discussion}

\section{Association of cane yield with other characters in sugarcane}

Simple correlation has been used to know the nature and extent of association between yield and its determinants. Selection for specific characters is known to result in correlated response in certain other characters (Falconer, 1964). Generally, plant breeders make selection for one or two attributes at a time, and then it becomes important to know the effect on other characters.

In the present investigation, genotypic and phenotypic correlation coefficients (Tables 1 and $2 \&$ Figs. 2 and 3) indicated that cane yield (t/ha) had significant and positive correlation with germination percentage at 45 DAP, number of shoots at 120 DAP (000/ha), number of shoots at $240 \mathrm{DAP}$ (000/ha), number of millable cane at harvest (000/ha), sugar yield ( $t /$ ha), plant height at 240 DAP, plant height at 360 DAP, single cane weight, cane diameter at harvest leaf area index before waterlogging, leaf area index 30 days after waterlogging, leaf area index 60 days after waterlogging, number of fully emerged leaves at 30 days after waterlogging and number of fully emerged leaves at 60 days after waterlogging with cane yield. Thus, these traits play a greater role as important contributing characters for higher cane yield. Whereas, purity percentage at 10 months stage, purity at 12 months stage, pol percentage at 10 months stage, brix percentage at 10 months stage, number of nodes with aerial roots, plants height at $150 \mathrm{DAP}$, plants height at 240 DAP and CCS percentage at 10 months stage showed non-significant and positive association with cane yield, but brix percentage at 12 months stage, pol percentage at 12 months stage and CCS percentage at 12 months stage showed non-significant and negative association with cane yield. Hence, simultaneous improvement of these characters is far from reality. Similar results were found by Chaudhary and Joshi (2005), they reported positive and highly significant correlation of cane yield with single cane weight, stalk length, millable cane number and cane diameter. Sanghera et al., (2015) reported significant and positive correlation of cane yield with number of millable cane at 10 months, stalk length, single cane weight, cane diameter and germination percentage at 45 DAP indicating the importance of these traits to be involved in selection criteria. Tena et al., (2016) reported that cane yield showed strong positive and highly significant correlation with number of millable cane, single cane weight, stalk height and sugar yield. Tadesse et al., (2014) found that cane yield was positively and highly significantly correlated with single cane weight and number of millable cane while positive and significantly correlated with cane diameter. Singh et al., (2005) observed that number of millable cane, shoot at 120 DAP, single cane weight, germination percentage at 45 DAP and cane height were significantly and positively correlated with cane yield. Singh $e t$ al., (2003) reported that brix percentage and purity percentage showed negative and nonsignificant correlation with cane yield.

Ahmed et al., (2010) observed positive correlation between cane yield and number of millable stalk, stalk height, single stalk weight indicating improvement in any of these characters may result in positive influence on other characters while negative association of cane yield with pol percentage and purity percentage was also noticed in this study. Kumar and Kumar (2014) reported that the cane yield was positively and significantly correlated with germination percentage, number of shoots, number of millable canes, stalk diameter, stalk length and stalk weight. Chandrakant (2007) revealed that number of shoots at 120 DAP, number of millable cane at harvest and single cane weight were had consistent trend of significant association with cane yield. 
Table.1 Genotypic correlation coefficient of twenty-five traits with cane yield of sugarcane clones under waterlogging condition

\begin{tabular}{|c|c|c|c|c|c|c|c|c|c|c|c|c|c|c|c|c|c|c|c|c|c|c|c|c|}
\hline Traits & $\mathrm{X} 1$ & $\mathrm{X} 2$ & $\mathbf{X 3}$ & $\mathrm{X} 4$ & X5 & $\mathrm{X} 7$ & $\mathrm{X8}$ & X9 & $\mathbf{X 1 0}$ & X11 & $\mathrm{X12}$ & $\mathrm{X13}$ & X14 & X15 & $\mathrm{X16}$ & X17 & $\mathbf{X 1 8}$ & $\mathbf{X 1 9}$ & $\mathbf{X 2 0}$ & $\mathrm{X} 21$ & $\mathrm{X} 22$ & $\mathbf{X 2 3}$ & X24 & $\mathrm{X} 25$ \\
\hline X1 & 1.000 & & & & & & & & & & & & & & & & & & & & & & & \\
\hline $\mathrm{X} 2$ & 0.957 & 1.000 & & & & & & & & & & & & & & & & & & & & & & \\
\hline $\mathrm{X3}$ & 0.838 & 0.934 & 1.000 & & & & & & & & & & & & & & & & & & & & & \\
\hline $\mathrm{X} 4$ & 0.825 & 0.980 & 0.885 & 1.000 & & & & & & & & & & & & & & & & & & & & \\
\hline X5 & 0.579 & 0.761 & 0.641 & 0.892 & 1.000 & & & & & & & & & & & & & & & & & & & \\
\hline $\mathrm{X} 7$ & 0.217 & 0.130 & 0.260 & -0.101 & $\begin{array}{l}-0.062 \\
\end{array}$ & 1.000 & & & & & & & & & & & & & & & & & & \\
\hline X8 & 0.073 & 0.232 & 0.205 & 0.340 & 0.421 & -0.061 & 1.000 & & & & & & & & & & & & & & & & & \\
\hline X9 & 0.051 & -0.211 & 0.054 & -0.300 & -0.297 & 0.893 & 0.157 & 1.000 & & & & & & & & & & & & & & & & \\
\hline $\mathrm{X10}$ & -0.148 & 0.023 & 0.079 & 0.002 & 0.198 & 0.726 & 0.537 & 0.405 & 1.000 & & & & & & & & & & & & & & & \\
\hline X11 & 0.148 & 0.024 & 0.180 & $\begin{array}{l}-0.172 \\
\end{array}$ & -0.141 & 0.997 & -0.041 & 0.931 & 0.667 & 1.000 & & & & & & & & & & & & & & \\
\hline $\mathrm{X12}$ & $\begin{array}{l}-0.166 \\
\end{array}$ & -0.023 & 0.046 & -0.071 & 0.138 & 0.810 & 0.424 & 0.428 & 0.901 & 0.747 & 1.000 & & & & & & & & & & & & & \\
\hline X13 & $\begin{array}{l}-0.195 \\
\end{array}$ & -0.027 & -0.117 & $\begin{array}{l}-0.161 \\
\end{array}$ & 0.371 & 0.106 & -0.071 & -0.130 & 0.496 & 0.089 & 0.530 & 1.000 & & & & & & & & & & & & \\
\hline X14 & -0.039 & 0.100 & 0.537 & 0.098 & -0.013 & -0.071 & 0.012 & -0.494 & 0.288 & -0.179 & 0.331 & 0.579 & 1.000 & & & & & & & & & & & \\
\hline $\mathrm{X} 15$ & 0.689 & 0.760 & 0.758 & 0.783 & 0.529 & -0.188 & $\begin{array}{l}-0.447 \\
\end{array}$ & -0.251 & -0.348 & -0.230 & \begin{tabular}{c|}
-0.236 \\
\end{tabular} & -0.065 & 0.038 & 1.000 & & & & & & & & & & \\
\hline X16 & 0.639 & 0.639 & 0.760 & 0.709 & 0.406 & 0.015 & -0.525 & -0.260 & $\begin{array}{l}-0.417 \\
\end{array}$ & -0.069 & -0.306 & -0.157 & $\begin{array}{l}-0.007 \\
\end{array}$ & 0.927 & 1.000 & & & & & & & & & \\
\hline X17 & 0.055 & 0.300 & 0.117 & 0.461 & 0.791 & -0.303 & 0.453 & -0.424 & 0.129 & -0.351 & 0.055 & 0.783 & 0.043 & 0.120 & -0.093 & 1.000 & & & & & & & & \\
\hline X18 & 0.050 & 0.197 & -0.034 & 0.256 & 0.775 & -0.162 & 0.242 & -0.371 & 0.014 & -0.214 & -0.038 & 0.680 & 0.056 & 0.191 & 0.024 & .951 & 1.000 & & & & & & & \\
\hline X19 & 0.432 & 0.494 & 0.374 & 0.705 & 0.793 & $\begin{array}{l}-0.462 \\
\end{array}$ & 0.082 & $\begin{array}{l}-0.603 \\
\end{array}$ & $\begin{array}{l}-0.076 \\
\end{array}$ & -0.522 & -0.100 & 0.608 & 0.038 & 0.705 & 0.603 & 0.796 & 0.841 & 1.000 & & & & & & \\
\hline $\mathbf{X 2 0}$ & 0.301 & 0.460 & 0.482 & 0.757 & 0.808 & -0.372 & 0.247 & $\begin{array}{c}-0.570 \\
\end{array}$ & $\begin{array}{c}-0.016 \\
\end{array}$ & -0.456 & -0.071 & 0.517 & 0.246 & 0.715 & 0.553 & 0.708 & 0.659 & 0.901 & 1.000 & & & & & \\
\hline $\mathbf{X 2 1}$ & 0.431 & 0.487 & 0.608 & 0.786 & 0.661 & -0.422 & 0.200 & -0.285 & $\begin{array}{c}-0.141 \\
\end{array}$ & -0.423 & -0.200 & 0.221 & 0.182 & 0.761 & 0.653 & 0.394 & 0.149 & 0.816 & 0.960 & 1.000 & & & & \\
\hline $\mathrm{X} 22$ & 0.342 & 0.297 & 0.525 & 0.429 & 0.540 & 0.300 & 0.027 & $\begin{array}{c}-0.051 \\
\end{array}$ & 0.540 & 0.201 & 0.563 & 0.954 & 0.538 & 0.476 & 0.344 & 0.342 & 0.259 & 0.476 & 0.561 & 0.483 & 1.000 & & & \\
\hline $\mathrm{X} 23$ & 0.458 & 0.445 & 0.639 & 0.719 & 0.855 & 0.508 & 0.165 & -0.199 & 0.704 & 0.324 & 0.695 & 0.965 & 0.431 & 0.674 & 0.541 & 0.487 & 0.223 & 0.746 & 0.767 & 0.508 & 0.917 & 1.000 & & \\
\hline X24 & -0.111 & 0.037 & 0.107 & 0.037 & 0.221 & 0.680 & 0.603 & 0.383 & 0.998 & 0.621 & 0.991 & 0.441 & 0.245 & -0.410 & -0.473 & 0.152 & 0.010 & $\begin{array}{l}-0.077 \\
\end{array}$ & 0.001 & -0.122 & 0.503 & 0.670 & 1.000 & \\
\hline $\mathrm{X} 25$ & 0.125 & -0.012 & 0.152 & -0.194 & -0.166 & 0.994 & -0.026 & 0.944 & 0.651 & 0.999 & 0.728 & 0.079 & -0.214 & -0.245 & $\begin{array}{c}-0.099 \\
\end{array}$ & -0.366 & -0.234 & -0.542 & -0.482 & -0.420 & 0.169 & 0.263 & 0.606 & 1.000 \\
\hline X6 & 0.521 & 0.723 & 0.564 & 0.870 & 0.979 & -0.264 & 0.427 & $\begin{array}{c}-0.466 \\
\end{array}$ & 0.054 & $\begin{array}{l}-0.338 \\
\end{array}$ & $\begin{array}{c}-0.025 \\
\end{array}$ & 0.330 & 0.027 & 0.545 & 0.392 & 0.840 & 0.802 & 0.876 & 0.865 & 0.707 & 0.456 & 0.740 & 0.087 & -0.360 \\
\hline
\end{tabular}

X1- Germination Percentage at 45 DAP

X2- Number of Shoots at 120 DAP

X3- Number of Shoots at 240 DAP

X4- Number of Millable Canes at Harvest (000/ha)

X5- Sugar Yield (t/ha)

X6- Cane yield (t/ha)

X7- Brix \% at 12 Months Stage

X8- Purity $\%$ at 10 Months Stage

X9- Purity \% at 12 Months Stage

X10- Pol \% at 10 Months Stage

$\mathrm{X} 11$ - Pol \% at 12 Months Stage

$\mathrm{X} 12-\mathrm{Brix} \%$ at 10 Months Stage
X13- Number of Nodes with Aerial Roots

X14- Plant Height at 150 DAP $(\mathrm{cm})$

X15- Plant Height at 240 DAP (cm)

X16- Plant Height at 360 DAP $(\mathrm{cm})$

X17- Single Cane Weight $(\mathrm{kg})$

X18- Cane Diameter at Harvest (cm)

X19- Leaf Area Index Before Waterlogging

X20- Leaf Area Index at 30 Days After Waterlogging

X21 - Leaf Area Index at 60 Days After Waterlogging

X22- Number of Fully Emerged Leaves at 30 Days After Waterlogging

X23- Number of Fully Emerged Leaves at 60 Days After Waterlogging

X24- CCS \% at 10 Months Stage

$\mathrm{X} 25-\mathrm{CCS} \%$ at 12 Months Stage 
Table.2 Phenotypic correlation coefficient of twenty-five traits with cane yield of sugarcane clones under waterlogging condition

\begin{tabular}{|c|c|c|c|c|c|c|c|c|c|c|c|c|c|c|c|c|c|c|c|c|c|c|c|c|}
\hline Traits & $\mathrm{X1}$ & $\mathrm{X} 2$ & $\mathbf{x} 3$ & $\mathrm{X} 4$ & $\mathrm{X5}$ & $\mathrm{X} 7$ & $\mathrm{X8}$ & X9 & $\mathrm{X10}$ & $\mathrm{X11}$ & $\mathrm{X12}$ & $\mathrm{X13}$ & $\mathrm{X} 14$ & $\mathrm{X} 15$ & X16 & $\mathrm{X17}$ & $\mathrm{X18}$ & X19 & $\mathrm{X} 20$ & $\mathrm{X} 21$ & $\mathrm{X} 22$ & $\mathrm{X} 23$ & $\mathrm{X} 24$ & $\mathrm{X} 25$ \\
\hline $\mathrm{X1}$ & 1.000 & & & & & & & & & & & & & & & & & & & & & & & \\
\hline $\mathrm{X} 2$ & $0.584 * *$ & 1.000 & & & & & & & & & & & & & & & & & & & & & & \\
\hline $\mathrm{X3}$ & $0.623^{* * *}$ & $0.593 * *$ & 1.000 & & & & & & & & & & & & & & & & & & & & & \\
\hline $\mathrm{X} 4$ & $0.601^{* *}$ & $0.532 * *$ & $0.610 * *$ & 1.000 & & & & & & & & & & & & & & & & & & & & \\
\hline $\mathrm{X5}$ & 0.400 *** & $0.553 * *$ & $0.514 * *$ & $0.782 * *$ & 1.000 & & & & & & & & & & & & & & & & & & & \\
\hline $\mathrm{X7}$ & 0.013 & 0.024 & 0.171 & -0.018 & 0.174 & 1.000 & & & & & & & & & & & & & & & & & & \\
\hline $\mathrm{X8}$ & 0.017 & 0.095 & 0.048 & 0.129 & 0.309 & 0.173 & 1.000 & & & & & & & & & & & & & & & & & \\
\hline X9 & -0.043 & 0.106 & -0.016 & -0.262 & -0.068 & $0.394 * *$ & 0.205 & 1.000 & & & & & & & & & & & & & & & & \\
\hline $\mathrm{X10}$ & -0.039 & $\begin{array}{l}-0.049 \\
\end{array}$ & 0.081 & 0.073 & 0.274 & $0.528 * *$ & $0.624 * *$ & 0.142 & 1.000 & & & & & & & & & & & & & & & \\
\hline $\mathrm{X} 11$ & \begin{tabular}{|c|}
-0.016 \\
\end{tabular} & 0.036 & 0.119 & \begin{tabular}{|c|}
-0.111 \\
\end{tabular} & 0.118 & $0.958 * *$ & 0.208 & $0.629 * *$ & $0.501^{* *}$ & 1.000 & & & & & & & & & & & & & & \\
\hline $\mathrm{X12}$ & $\begin{array}{l}-0.055 \\
\end{array}$ & \begin{tabular}{|c|}
-0.073 \\
\end{tabular} & 0.085 & 0.061 & 0.226 & $0.554 * *$ & $0.409 * *$ & 0.082 & $0.963^{* * *}$ & $0.505 * *$ & 1.000 & & & & & & & & & & & & & \\
\hline $\mathrm{X} 13$ & $\begin{array}{l}-0.008 \\
\end{array}$ & -0.072 & -0.054 & 0.070 & 0.131 & 0.010 & $\begin{array}{l}-0.105 \\
\end{array}$ & -0.185 & 0.140 & $\begin{array}{l}-0.054 \\
\end{array}$ & 0.204 & 1.000 & & & & & & & & & & & & \\
\hline X14 & -0.080 & -0.020 & 0.210 & 0.006 & 0.112 & 0.213 & 0.120 & 0.041 & 0.227 & 0.187 & 0.213 & 0.217 & 1.000 & & & & & & & & & & & \\
\hline $\mathrm{X} 15$ & 0.231 & 0.265 & $0.286^{*}$ & $0.432 * *$ & $0.394 * *$ & 0.159 & 0.023 & -0.074 & 0.016 & 0.106 & -0.021 & -0.022 & 0.273 & 1.000 & & & & & & & & & & \\
\hline X16 & $0.296^{*}$ & 0.185 & 0.245 & $0.444 * *$ & $0.318^{*}$ & 0.124 & -0.146 & -0.052 & -0.079 & 0.084 & -0.077 & -0.168 & 0.224 & $0.878^{* * *}$ & 1.000 & & & & & & & & & \\
\hline X17 & 0.051 & $0.325 *$ & 0.129 & 0.223 & $0.699 * *$ & $\begin{array}{l}-0.145 \\
\end{array}$ & 0.278 & $\begin{array}{l}-0.127 \\
\end{array}$ & 0.137 & $\begin{array}{l}-0.157 \\
\end{array}$ & 0.072 & 0.184 & 0.090 & 0.097 & \begin{tabular}{|c|}
-0.039 \\
\end{tabular} & 1.000 & & & & & & & & \\
\hline X18 & 0.094 & 0.136 & -0.042 & 0.196 & $0.389 * *$ & -0.190 & 0.077 & -0.137 & -0.041 & -0.202 & -0.073 & $0.367^{*}$ & -0.031 & 0.204 & 0.177 & $0.549^{* * *}$ & 1.000 & & & & & & & \\
\hline X19 & 0.216 & $0.347^{*}$ & 0.266 & $0.452 * *$ & $0.553^{* * *}$ & -0.135 & 0.036 & $-0.287^{*}$ & -0.082 & -0.201 & -0.097 & 0.200 & 0.098 & $0.414^{* *}$ & $0.330^{*}$ & $0.458^{* * *}$ & $0.409^{* *}$ & 1.000 & & & & & & \\
\hline $\mathrm{X} 20$ & 0.267 & 0.248 & $0.298^{*}$ & $0.508^{* *}$ & $0.493 * *$ & $-0.318^{*}$ & 0.109 & $-0.394 * *$ & -0.027 & $-0.390 * *$ & \begin{tabular}{|c|}
-0.053 \\
\end{tabular} & 0.225 & 0.110 & $0.312^{*}$ & 0.275 & $0.412 * *$ & $0.302 *$ & $0.764 * *$ & 1.000 & & & & & \\
\hline $\mathrm{X} 21$ & $0.350 *$ & $0.352^{*}$ & $0.353^{*}$ & $0.491 * *$ & $0.371^{* * *}$ & $-0.315^{*}$ & 0.031 & $\begin{array}{l}-0.269 \\
\end{array}$ & -0.138 & $-0.355^{*}$ & \begin{tabular}{|l|}
-0.153 \\
\end{tabular} & 0.145 & -0.003 & 0.284 & 0.179 & 0.240 & 0.201 & $0.554 * *$ & $0.773 * *$ & 1.000 & & & & \\
\hline $\mathrm{X} 22$ & 0.134 & 0.160 & 0.324* & 0.272 & $0.355^{*}$ & 0.230 & $\begin{array}{l}-0.120 \\
\end{array}$ & -0.030 & 0.217 & 0.182 & $0.299 *$ & $0.425 * *$ & $0.302^{*}$ & 0.195 & 0.096 & 0.177 & 0.007 & $0.379 * *$ & $0.338 *$ & $0.330^{*}$ & 1.000 & & & \\
\hline $\mathrm{X} 23$ & 0.251 & $0.389 * *$ & $0.413 * *$ & $0.353^{*}$ & $0.432^{* * *}$ & 0.102 & -0.115 & -0.016 & 0.118 & 0.069 & 0.193 & 0.245 & 0.092 & $0.322^{*}$ & 0.226 & 0.294* & 0.364* & $0.448 * *$ & $0.361^{*}$ & $0.378^{* * *}$ & $0.611^{* *}$ & 1.000 & & \\
\hline $\mathrm{X24}$ & $\begin{array}{l}-0.034 \\
\end{array}$ & -0.041 & 0.073 & 0.090 & $0.292^{*}$ & $0.503^{* *}$ & $0.694 * *$ & 0.171 & $0.993 * *$ & $0.488^{* * *}$ & $0.930^{* * *}$ & 0.110 & 0.219 & 0.024 & -0.083 & 0.153 & $\begin{array}{l}-0.024 \\
\end{array}$ & -0.092 & $\begin{array}{l}-0.029 \\
\end{array}$ & -0.127 & 0.189 & 0.099 & 1.000 & \\
\hline X25 & -0.025 & 0.038 & 0.100 & $\begin{array}{l}-0.141 \\
\end{array}$ & 0.095 & $0.923 * *$ & 0.218 & $0.701^{* * *}$ & $0.482 * *$ & $0.995^{* * *}$ & $0.478^{* * *}$ & -0.074 & 0.174 & 0.082 & 0.065 & -0.160 & $\begin{array}{l}-0.204 \\
\end{array}$ & $\begin{array}{l}-0.223 \\
\end{array}$ & $-0.407^{* * *}$ & $-0.361 *$ & 0.160 & 0.056 & $0.473^{* * *}$ & 1.000 \\
\hline X6 & $0.403^{* * *}$ & $0.543 * *$ & $0.473 * *$ & $0.800 * *$ & $0.961^{* * *}$ & $\begin{array}{l}-0.087 \\
\end{array}$ & 0.258 & $\begin{array}{l}-0.247 \\
\end{array}$ & 0.139 & $\begin{array}{l}-0.157 \\
\end{array}$ & 0.090 & 0.153 & 0.058 & $0.358 *$ & 0.286* & $0.751^{* * *}$ & $0.454 * *$ & $0.617 * *$ & $0.603 * *$ & $0.463 * *$ & 0.296* & $0.400 * *$ & 0.160 & $\begin{array}{l}-0.179 \\
\end{array}$ \\
\hline
\end{tabular}

* Significant at $5 \%$,

** Significant at $1 \%$

X1- Germination Percentage at 45 DAP

X2- Number of Shoots at 120 DAP

X3- Number of Shoots at 240 DAP

X4- Number of Millable Canes at Harvest (000/ha)

X5- Sugar Yield (t/ha)

X6- Cane yield (t/ha)

X7- Brix \% at 12 Months Stage

X8- Purity \% at 10 Months Stage

X9- Purity $\%$ at 12 Months Stage

$\mathrm{X} 10-\mathrm{Pol} \%$ at 10 Months Stage

$\mathrm{X} 11-\mathrm{Pol} \%$ at 12 Months Stage

X12- Brix \% at 10 Months Stage

X13- Number of Nodes with Aerial Roots

X14- Plant Height at 150 DAP $(\mathrm{cm})$

X15- Plant Height at 240 DAP $(\mathrm{cm})$

X16- Plant Height at 360 DAP $(\mathrm{cm})$

X17- Single Cane Weight (kg)

X18- Cane Diameter at Harvest $(\mathrm{cm})$

X19- Leaf Area Index Before Waterlogging

X20- Leaf Area Index at 30 Days After Waterlogging

X21 - Leaf Area Index at 60 Days After Waterlogging

X22- Number of Fully Emerged Leaves at 30 Days After Waterlogging

X23- Number of Fully Emerged Leaves at 60 Days After Waterlogging

$\mathrm{X} 24-\mathrm{CCS} \%$ at 10 Months Stage

$\mathrm{X} 25-\mathrm{CCS} \%$ at 12 Months Stage 


\section{Int.J.Curr.Microbiol.App.Sci (2017) 6(10): 2331-2339}

Fig.1 Meteorological data recorded monthly during cropping season 2016-17

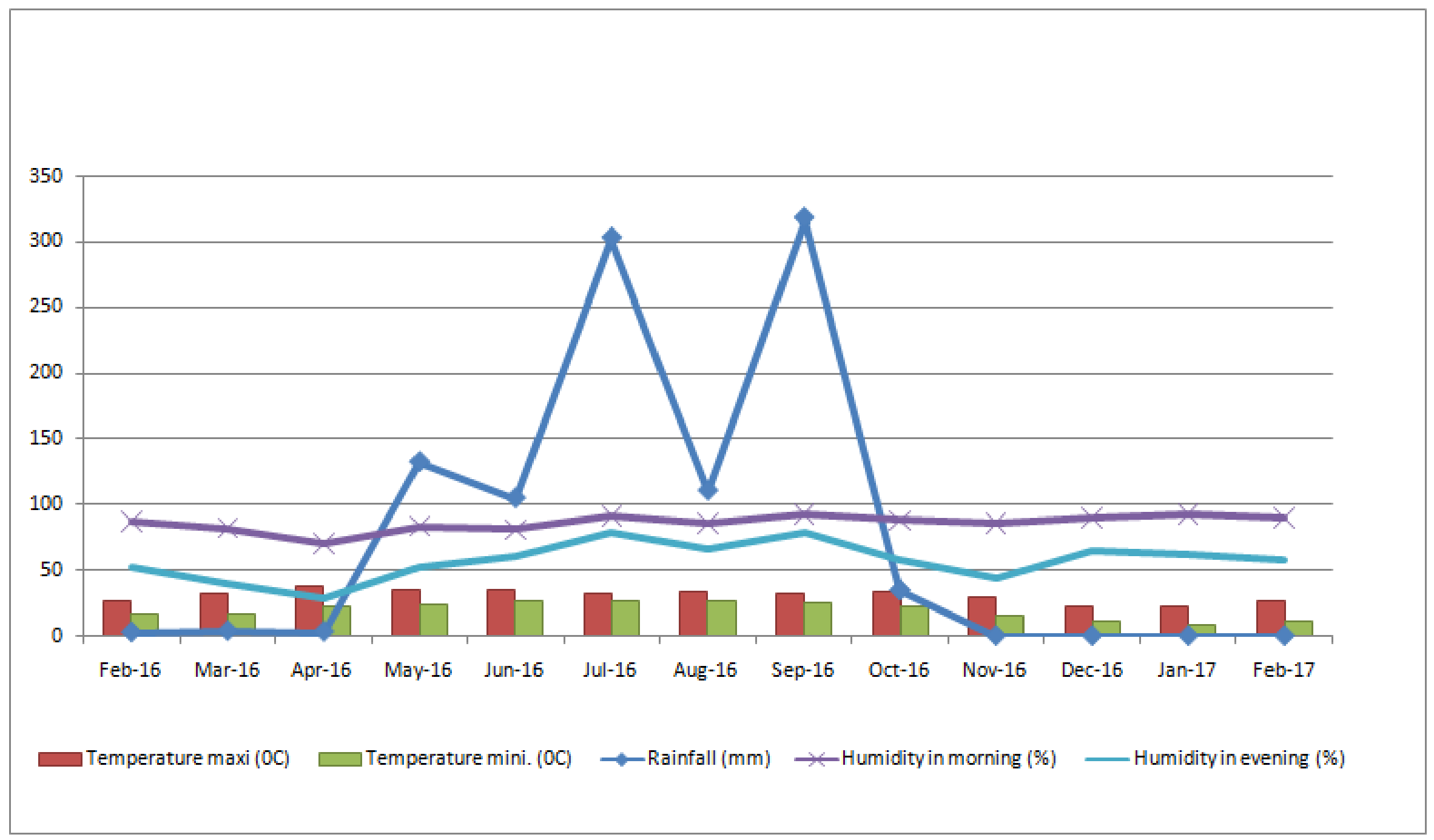

Source: Dept. of Agro meteorology, D.R.P.C.A.U., Pusa, Samastipur, Bihar 
Murthy (2007) reported significant and positive association of cane yield with single cane weight and number of millable cane indicating the importance of these traits in selection of sugarcane genotype for higher sugar yield and cane yield.

Association among juice quality and with other cane characters

With regards to correlation coefficient among the quality traits, there was highly significant positive association among purity percentage at 10 and 12 months stage, brix percentage at 10 and 12 months stage, CCS percentage at 10 and 12 months stage, pol percentage at 10 and 12 months stage as expected indicating the interrelationship among themselves. Thus any of these juice quality traits could be considered for selection leading to the simultaneous improvement in the remaining quality traits. Similar results were reported by Kang et al., (1983), Sreekumar et al., (1994), Tena et al., (2016), Thangavelu (2011) and Thippeswamy et al., (2003).

\section{Correlation among yield attributing characters}

Present investigation indicated the germination percentage at 45 DAP, number of shoot at 120 and 240 DAP, number of millabile canes at harvest (000/ha), sugar yield (CCS t/ha), cane yield and leaf area index at 60 days after waterlogging showed either significant or highly significant positive association among themselves. Thus the interrelationship among these traits indicated that improvement in one attributes would certainly leads to the improvement in other traits in desired direction. These results are in conformity with the observation of Masri et al., (2015). Similar result were also reported by Khan et al., (2013), Verma et al., (1999). Leaf area index before waterlogging exhibited significant negative association with purity percentage at 12 months stage and leaf area index at 30 and 60 days after waterlogging showed significant negative association with brix CCS and purity percentage at 12 months stage respectively. Hence, simultaneous improvement of these characters is far from reality.

Single cane weight had significant and positive correlation with number of shoots at 120 DAP, sugar yield (CCS t/ha), cane diameter and leaf area index before waterlogging. The leaf area index before waterlogging had significant and positive correlation with number of shoot at 120 DAP, number of millable canes at harvest (000/ha), sugar yield (CCS t/ha), plat height at 240 DAO, plant height at 360 DAP, single cane weight, cane diameter, leaf area index at 30 days after waterlogging, leaf area index at 60 days after waterlogging, number of fully emerged leaves at 30 and 60 days after waterlogging respectively. Thus any of these yield attributing traits could be considered for selection leading to the simultaneous improvement in yield attributing traits.

Cane yield ( $\mathrm{t} / \mathrm{ha}$ ) had significant and positive correlation with germination percentage at 45 DAP, number of shoots at 120 DAP (000/ha), number of shoots at $240 \mathrm{DAP}(000 / \mathrm{ha})$, number of millable canes at harvest (000/ha), sugar yield ( $\mathrm{t} / \mathrm{ha}$ ), plant height at 240 DAP, plant height at 360 DAP, single cane weight, cane diameter at harvest, leaf area index before waterlogging, leaf area index 30 days after waterlogging, leaf area index 60 days after waterlogging, number of fully emerged leaves at 30 days after waterlogging and number of fully emerged leaves at 60 days after waterlogging with cane yield at both genotypic and phenotypic level. With regards to correlation coefficient among the quality traits, there was highly significant positive association among purity percentage at 10 and 12 months stage, brix percentage at 10 and 12 
months stage, CCS percentage at 10 and 12 months stage, pol percentage at 10 and 12 months stage. Correlation among yield attributing characters indicated that the germination percentage at $45 \mathrm{DAP}$, number of shoot at 120 and 240 DAP, number of millabile canes at harvest (000/ha), sugar yield (CCS t/ha), cane yield and leaf area index at 60 days after waterlogging showed either significant or highly significant positive association among themselves.

\section{References}

Ahmed, A.O., Obeid, A. and Dafallah, B. 2010. The influence of characters association on behavior of sugarcane genotypes (Saccharum spp.) for cane yield and juice quality. World $J$. of Agricultural Sciences. 6(2): 207-211.

Al-Jibouri, H.A., Miller, P.A. and Robinson, H.F. 1958. Genotypic and environmental variances in an upland cotton cross of interspecific origin. Agron. J. 50: 633-637.

Chandrakant., Ravikant and Singh, P.K. 2007. Screening of promising clones in early clonal generations from pool of Saccharum complex hybrids. Ann. Agric. Res. New series. 28(1): 37-43.

Choudhary, R.R., and Joshi, B.K. 2005. Correlation and Path coefficient in sugarcane. Nepal Agric. Res. J. 6: 2427.

De Sousa-V. O., and M. B. Scott. 2005. Interrelationship of cane yield components and their utility in sugarcane family selection: Path coefficient analysis. INCI. 30(2):93-96.

Falconer, D.S., 1964. Introduction to Quantitative Genetics, Oliver and Boyd, London. pp 365.

Falconer, D.S., 1981. Introduction to Quantitative Genetics, 2nd edition, Longman Group Ltd., Longman House, Harrow, England. pp 350.
Furtado, M.R., C.D. Cruz, A.A. Cardoso, A.D. Fernandes and L.A. Peternelli. 2002. Análise de trilha do rendimento do feijoeiro e seus componentes primários em monocultivo e em consórcio com a cultura do milho. Ciência Rural. 32: 217-220.

Johnson, H.W., Robinson, H.F. and Comstock, R.E. 1955. Estimates of genetic and environmental variability in soybean. Agron. J. 47: 314-318.

Kang, M.S., Miller, J.D. and Tai, P.Y.P. 1983. Genetic and phenotypic path analysis and heritability in sugarcane. Crop Sci. 23: 643-647.

Khan, I. A., Seema, N., Raza, S., Yasmine, S. and Bibi, S. 2013. Environmental interactions of sugarcane genotypes and yield stability analysis of sugarcane. Pak. J. Bot. 45(5): 1617-1622.

Kumar, S., and Kumar, D. 2014. Correlation and path coefficient analysis in sugarcane germplasm under subtropics. African Journal of Agricultura Research. 9(1): 148-153.

Masri, M.I., Shaban, A., Hennawy, H.H.E., Taib, A.B.A.E., and Lail, F.F.B.A.E. 2015. Phenotypic and genotypic correlations and path coefficient analysis in sugarcane at first clonal selection stage. Egypt. J. Pla. nt Breed. 19(2): $297-321$

Murthy, N., 2007. Genetic variability and character association for yield and quality parameters in sugarcane. $J$. Maharashtra agric. Univ. 32(3): 343346.

Panse, V.G., and Sukhatme, P.V. 1967. Statistical method for agricultural worker, $2^{\text {nd }}$ Edt. ICAR, New Delhi. pp. 152-157.

Sanghera, G.S., Tyagi, V., Kumar, R., Thind, K.S. and Sharma, B. 2015. Genetic variability, association and their dissection through path analysis for cane yield and its component traits in 
early maturing sugarcane clones. Journal of Science. 5(1): 28-34.

Singh, A., Bhatnagar, P.K., Khan, A.Q. and Shrotria, P.K. 2003. Association of quality character with cane and commercial cane sugar yield in sugarcane. Sugar Tech. 5(3): 197-198.

Singh, J.R.P., Kamat, D.N. and Kumar, A. 2005. Correlation studies in sugarcane under saline condition. Indian Sugar. 55(1): 19-21.

Sreekumar, K., Jessy, K., Mathew, T., Alexander, D. and Santhakumari, S. 1994. Variability, heritability and correlation studies on the yield and quality characters of sugarcane. Indian Sugar. 243-249.

Stevenson, G.C., 1965. Genetics and Breeding of Sugarcane. Tropical Science Series. Longman's Green and Co., London.

Tadesse, F., and Dilnesaw, Z. 2014. Genetic Variability, Heritability and Character Association of Twelve Sugar Cane Varieties in Finchaa Sugar Estate West Wolega Zone Oromia Region of
Ethiopia. Int. J. Adv. Res. Biol. Sci. 1(7): 131-137.

Tena, E., Mekbib, F., Ayana, A. 2016. Heritability and Correlation among Sugarcane (Saccharum spp.) Yield and Some Agronomic and Sugar Quality Traits in Ethiopia. American Journal of Plant Sciences. 7: 1453-1477.

Thangavelu, S., 2011. Determination of commercial cane sugar. Cooperative Sugar. 42(10): 53-55.

Thippeswamy, S., Kajjidoni, S.T., Salimath, P.M. and Goud, J.V. 2003. Correlation and path analysis for cane yield, juice quality and their component traits in sugarcane. Sugar Tech. 5(1/2): 65-72.

Tyagi, V.K., S. Sharma and S.B. Bhardwaj. 2012. Pattern of association among cane yield, sugar yield and their components in sugarcane (Saccharum officinarum L.). J. Agric. Res. 50(1): 29-38.

Verma, P.S., Pal, S, and Karma, N.K. 1999. Genetic variability and correlation studies in sugarcane. Indian Sugar. 49(2): 125-128.

\section{How to cite this article:}

Bal Krishna and Dharm Nath Kamat. 2017. Character Association among Cane Yield and their Component Traits in Sugarcane under Waterlogged Condition. Int.J.Curr.Microbiol.App.Sci. 6(10): 2331-2339. doi: https://doi.org/10.20546/ijcmas.2017.610.275 